\author{
Antônio F. B. A. Prado \\ Member, $A B C M$ \\ prado@dem.inpe.br \\ Instituto Nacional de Pesquisas Espaciais - INPE \\ Av. dos Astronautas 1758 \\ Caixa Postal 515 \\ 12227-010 São José dos Campos, SP, Brazil
}

\section{Orbital Control of a Satellite Using the Gravity of the Moon}

Abstract. In this paper, a study is made in the problem of the orbital control of an Earth's satellite using the gravity of the Moon. The main objective is to study a technique to decrease the fuel consumption of a plane change maneuver to be performed in a satellite that is in orbit around the Earth. The main idea of this approach is to send the spacecraft to the Moon using a single impulsive maneuver, use the gravity field of the Moon to make the desired plane change of the trajectory, and then return the spacecraft to its nominal semi-major axis and eccentricity using a bi-impulsive Hohmann type maneuver. The spacecraft is assumed to start in a circular orbit in the plane of the lunar orbit around the Earth and the goal is to put it in a similar orbit that differs from the initial orbit only by the inclination. A description of the close approach maneuver is made in the threedimensional space. Analytical equations based in the patched conics approximation are used to calculate the variation in velocity, angular momentum, energy and inclination of the spacecraft that realizes this maneuver. Then, several simulations are made to evaluate the savings involved.

Keywords: Astrodynamics, orbital maneuver, swing-by

\section{Introduction}

The Swing-By maneuver is a very popular technique used to decrease the fuel expenditure in space missions. The standard maneuver uses a close approach with a celestial body to modify the velocity, energy and angular momentum of the spacecraft. The literature shows several applications of the swing-by technique. Some of them can be found in Swenson (1992), that studied a mission to Neptune using swing-bys to gain energy to accomplish the mission; Weinstein (1992), that made a similar study for a mission to Pluto; Farquhar and Dunham (1981), that formulated a mission to study the Earth's geomagnetic tail; Farquhar, Muhonen and Church (1985), Efron, Yeomans, and Schanzle (1985) and Muhonen, Davis, and Dunham (1985), that planned the mission ISEE-3/ICE; Flandro (1966), that made the first studies for the Voyager mission; Byrnes and D'Amario (1982), that design a mission to flyby the comet Halley; D'Amario, Byrnes and Stanford (1981 and 1982) that studied multiple flyby for interplanetary missions; Marsh and Howell (1988) and Dunham and Davis (1985) that design missions with multiple lunar swing-bys; Prado and Broucke (1994), that studied the effects of the atmosphere in a swing-by trajectory; Striepe and Braun (1991), that used a swing-by in Venus to reach Mars; Felipe and Prado (1999 and 2000), that studied numerically a swing-by in three dimensions, including the effects in the inclination; Prado (1996), that considered the possibility of applying an impulse during the passage by the periapsis; Prado and Broucke (1995), that classified trajectories making a swing-by with the Moon. The most usual approach to study this problem is to divide the problem in three phases dominated by the "two-body" celestial mechanics. Other models used to study this problem are the circular restricted three-body problem (like in Broucke (1988), Broucke and Prado (1993), and Prado (1993)) and the elliptic restricted three-body problem (Prado (1997)).

The goal of this paper is to use analytical equations for the variations of velocity, energy, angular momentum and inclination to find an economical strategy to change the inclination of the orbit of a spacecraft by using a close approach with the Moon. The idea is to replace the expensive impulse required to make the plane change by a series of three impulses: the first one used to send the spacecraft in a planar trajectory to the Moon, to use the swing-by to make a free plane change and then to use the two remaining impulses to make a
Hohmann type planar transfer to change the semi-major axis and eccentricity that resulted from the swing-by to the desired nominal values. This maneuver was used by Hughes to save the AsiaSat 3 satellite after a failure with the rocket Proton, that delivered the spacecraft in a wrong orbit. Another advantage of this approach is that it allows the possibility of studying the Moon, making it a double objective mission. This maneuver will be compared with a direct single impulsive maneuver. There are other maneuvers that consider more impulses, like the bi-elliptic transfer with plane change, but they are not considered in this paper. Among the several sets of initial conditions that can be used to identify uniquely one swing-by trajectory, the following five variables are used (see Fig. (1)): $V_{p}$, the velocity of the spacecraft at periapsis of the orbit around the secondary body; two angles ( $\alpha$ and $\beta$ ), that specify the direction of the periapsis of the trajectory of the spacecraft around the secondary body in a three-dimensional space; $r_{p}$ the distance from the spacecraft to the center of the secondary body in the moment of the closest approach to this secondary body (periapsis distance); $\gamma$, the angle between the velocity vector at periapsis and the intersection between the horizontal plane that passes by the periapsis and the plane perpendicular to the periapsis that holds $\vec{V}_{p}$.

Figure 1 also shows the sequence for this maneuver. It is assumed that the system has three bodies: a primary $\left(\mathbf{M}_{1}\right.$, the Earth) and a secondary $\left(\mathrm{M}_{2}\right.$, the Moon) bodies with finite masses that are assumed to be in circular orbits around their common center of mass and a third body with negligible mass (the spacecraft) that has its motion governed by the two other bodies. The spacecraft leaves the point $\mathrm{A}$, passes by the point $\mathrm{P}$ (the periapsis of the trajectory of the spacecraft in its orbit around $\mathrm{M}_{2}$ ) and goes to the point $\mathrm{B}$. The points $\mathrm{A}$ and $\mathrm{B}$ are chosen in a such way that the influence of the Moon at those two points can be neglected and, consequently, the energy can be assumed to remain constant after B and before A (the system follows the two-body celestial mechanics). The initial conditions are clearly identified in Fig. 1. The distance $r_{p}$ is not to scale, to make the figure easier to understand. The result of this maneuver is a change in velocity, energy, angular momentum and inclination in the Keplerian orbit of the spacecraft around the central body. 


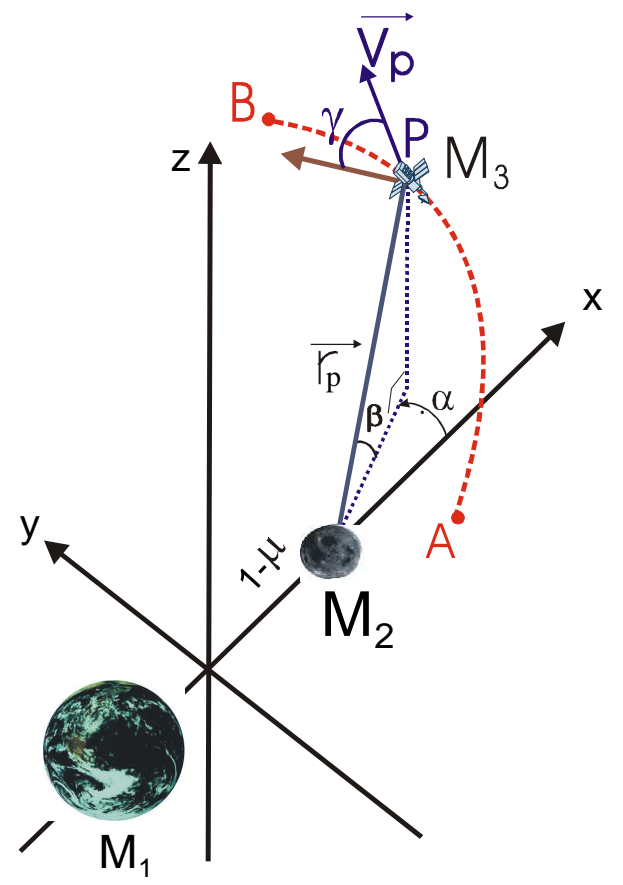

Figure 1. The swing-by in three dimensions.

\section{Mathematical model}

To start the description of the mathematical model used in this research, we briefly describe the orbital change of a single particle subjected to a close approach with the Moon under the "patchedconics" model. It is assumed that the particle is in an initial orbit around the Earth with given semi-major axis (a) and eccentricity (e) such that it crosses the orbit of the Moon and that this orbit is coplanar with the orbit of the Moon. Then, the swing-by is assumed to be planar for the equations developed below. The periapsis distance $\left(r_{p}\right)$ for the swing-by is assumed to be known. The canonical system of units is used, and it implies that: i) The unit of distance is the distance between the Earth and the Moon; ii) The angular velocity of the motion of the Earth and the Moon is assumed to be one; iii) The mass of the Moon is given by $\mu=\frac{m_{2}}{m_{1}+m_{2}}$ (where $\mathrm{m}_{1}$ and $\mathrm{m}_{2}$ are the real masses of the Earth and the Moon, respectively) and the mass of the Earth is $(1-\mu)$, so the total mass of the system is one; iv) The unit of time is defined such that the period of the motion of the Earth and the Moon is $2 \pi$; v) The gravitational constant is one.

The first step is to obtain the magnitude of the first impulse, the one used to send the spacecraft from its initial parking orbit around the Earth to the Moon. It is assumed that the initial orbit around the Earth is circular with semi-major axis $\mathrm{a}_{0}$ and that the transfer orbit is chosen to have semi-major axis a and eccentricity e. The minimum value for the semi-major axis that allows a transfer to the Moon is given by $\left(1+a_{0}\right) / 2$. In this way, the magnitude of the impulse required for this transfer and the eccentricity of the transfer orbit are given by:

$$
\Delta \mathrm{V}_{1}=\sqrt{\frac{2(1-\mu)}{\mathrm{a}_{0}}-\frac{(1-\mu)}{\mathrm{a}}}-\sqrt{\frac{(1-\mu)}{\mathrm{a}_{0}}}
$$

$$
\mathrm{e}=1-\frac{\mathrm{a}_{0}}{\mathrm{a}}
$$

Then, it is possible to calculate the magnitude of the velocity of the particle with respect to the Earth in the moment of the crossing with the Moon's orbit $\left(\mathrm{V}_{\mathrm{i}}\right)$, as well as the true anomaly of that point $(\theta)$. They come from

$$
\begin{gathered}
\mathrm{V}_{\mathrm{i}}=\sqrt{(1-\mu)\left(2-\frac{1}{\mathrm{a}}\right)} \\
\theta=\cos ^{-1}\left[\frac{1}{\mathrm{e}}\left(\mathrm{a}\left(1-\mathrm{e}^{2}\right)-1\right)\right]
\end{gathered}
$$

In those equations the fact that the distance between the Earth and the Moon is one was used and we took only the positive value of the true anomaly. Next, it is calculated the angle between the inertial velocity of the particle and the velocity of the Moon (the flight path angle $\gamma$ ), as well as the magnitude of the velocity of the particle with respect to the Moon in the moment that the approach starts $\left(\mathrm{V}_{\infty}\right)$. They are given by (assuming a counter-clock-wise orbit for the particle)

$$
\gamma=\tan ^{-1}\left[\frac{\mathrm{e} \sin \theta}{1+\mathrm{e} \cos \theta}\right]
$$

$$
\mathrm{V}_{\infty}=\sqrt{\mathrm{V}_{\mathrm{i}}^{2}+\mathrm{V}_{2}^{2}-2 \mathrm{~V}_{\mathrm{i}} \mathrm{V}_{2} \cos \gamma}
$$

Later we can use the fact that the velocity of the Moon around the Earth $\left(\mathrm{V}_{2}\right)$ is one. Figure 2 shows the vector addition used to derive the equations.

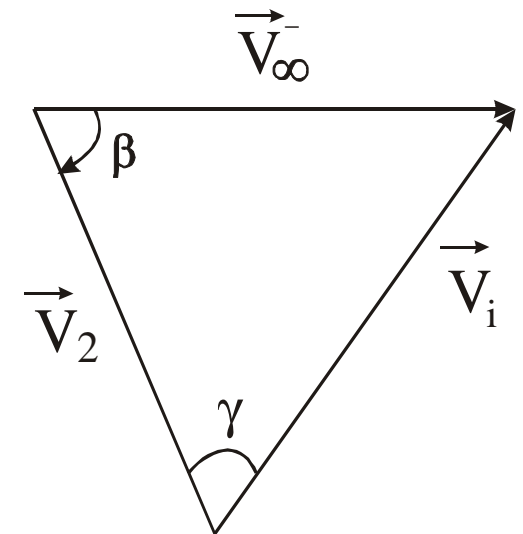

Figure 2. Vector addition during the close-approach.

The angle $\beta$ shown in Fig. 2 is given by

$$
\beta=\cos ^{-1}\left[-\frac{\mathrm{V}_{\mathrm{i}}^{2}-\mathrm{V}_{2}^{2}-\mathrm{V}_{\infty}^{-2}}{2 \mathrm{~V}_{2} \mathrm{~V}_{\infty}^{-}}\right]
$$

Those information allow us to obtain the turning angle $(2 \delta)$ of the particle around the Moon, from 


$$
\delta=\sin ^{-1}\left(1 / 1+\frac{r_{p} V_{\infty}^{2}}{\mu}\right)
$$

The angle of approach $(\alpha)$ has two values, depending if the particle is passing in front or behind Jupiter. These two values will be called $\alpha_{1}$ and $\alpha_{2}$. They are obtained from $\alpha_{1}=\pi+\beta+\delta$ and $\alpha_{2}=2 \pi+\beta-\delta$. In this paper only the first value is used, because the second one does not improve the efficiency of the swing-by maneuver.

Assuming that the spacecraft is coming from the same plane that the Moon orbits around the Earth and that the angle $\beta$ and the periapsis distance $r_{p}$ are used as control variables of the maneuver (they can be reached with maneuvers that has negligible consumption), we can obtain the following relation for the angle $\gamma$

$$
\gamma=\sin ^{-1}[-\operatorname{tg}(\delta) \operatorname{tg}(\beta)]
$$

Now it is necessary to have equations for the inertial velocities of the spacecraft before $\left(\mathbf{V}_{\mathbf{i}}\right)$ and after $\left(\mathbf{V}_{\mathbf{0}}\right)$ the close approach. They are (Prado, 2000)

$$
\begin{aligned}
\mathbf{V}_{\mathbf{i}}=\mathbf{V}_{\infty}^{-} & +\mathbf{V}_{2}=\mathrm{V}_{\infty} \sin \delta(\cos \beta \cos \alpha, \cos \beta \sin \alpha, \sin \beta)+ \\
+\mathrm{V}_{\infty} \cos \delta( & -\sin \gamma \sin \beta \cos \alpha-\cos \gamma \sin \alpha,-\sin \gamma \sin \beta \sin \alpha+ \\
& +\cos \gamma \cos \alpha, \cos \beta \sin \gamma)+\left(0, \mathrm{~V}_{2}, 0\right) \\
\mathbf{V}_{0}=\mathbf{V}_{\infty}^{+} & +\mathbf{V}_{2}=-\mathrm{V}_{\infty} \sin \delta(\cos \beta \cos \alpha, \cos \beta \sin \alpha, \sin \beta)+ \\
\mathrm{V}_{\infty} \cos \delta( & -\sin \gamma \sin \beta \cos \alpha-\cos \gamma \sin \alpha,-\sin \gamma \sin \beta \sin \alpha+ \\
& +\cos \gamma \cos \alpha, \cos \beta \sin \gamma)+\left(0, \mathrm{~V}_{2}, 0\right)
\end{aligned}
$$

From those equations, it is possible to obtain expressions for the variations in velocity, energy and angular momentum of the spacecraft. They are

$$
\Delta \mathbf{V}=\mathbf{V}_{\mathbf{0}}-\mathbf{V}_{\mathbf{i}}=-2 \mathbf{V}_{\infty} \sin \delta(\cos \alpha \cos \beta, \cos \beta \sin \alpha, \sin \beta)
$$

which implies that

$$
\begin{gathered}
\Delta \mathrm{V}=|\Delta \mathbf{V}|=2 \mathrm{~V}_{\infty} \sin \delta \\
\Delta \mathrm{E}=\frac{1}{2}\left(\mathrm{~V}_{0}^{2}-\mathrm{V}_{\mathrm{i}}^{2}\right)=-2 \mathrm{~V}_{2} \mathrm{~V}_{\infty} \cos \beta \sin \alpha \sin \delta
\end{gathered}
$$

For the angular momentum $(\mathbf{C})$ the results are:

$$
\begin{aligned}
& \mathbf{C}_{\mathbf{i}}=\mathbf{R} \times \mathbf{V}_{\mathbf{i}}=\mathrm{dV}_{\infty}\left(0,-\sin \beta \sin \delta+\cos \beta \cos \delta \sin \gamma, \frac{\mathrm{V}_{2}}{\mathrm{~V}_{\infty}}\right. \\
& +\cos \alpha \cos \delta \cos \gamma+\cos \beta \sin \alpha \sin \delta-\cos \delta \sin \alpha \sin \beta \sin \gamma)
\end{aligned}
$$

$$
\begin{aligned}
& \mathbf{C}_{\mathbf{0}}=\mathbf{R} \times \mathbf{V}_{\mathbf{0}}=\mathrm{dV}_{\infty}\left(0, \sin \beta \sin \delta-\cos \beta \cos \delta \sin \gamma, \frac{\mathrm{V}_{2}}{\mathrm{~V}_{\infty}}+\right. \\
& +\cos \alpha \cos \delta \cos \gamma-\cos \beta \sin \alpha \sin \delta-\cos \delta \sin \alpha \sin \beta \sin \gamma)
\end{aligned}
$$

Where $\mathbf{R}=(\mathrm{d}, 0,0)$ is the position vector of the Moon. Then

$$
\Delta \mathbf{C}=\mathbf{C}_{\mathbf{0}}-\mathbf{C}_{\mathbf{i}}=2 \mathrm{dV}_{\infty} \sin \delta(0, \sin \beta,-\cos \beta \sin \alpha)
$$

and

$$
|\Delta \mathbf{C}|=2 \mathrm{dV}_{\infty} \sin \delta\left(\cos ^{2} \beta \sin ^{2} \alpha+\sin ^{2} \beta\right)^{1 / 2}
$$

Using the definition of angular velocity $\omega=\frac{V_{2}}{d}$ it is possible to get

$$
\omega \Delta \mathrm{C}_{\mathrm{Z}}=-2 \mathrm{~V}_{2} \mathrm{~V}_{\infty} \cos \beta \sin \alpha \sin \delta=\Delta \mathrm{E}
$$

The inclination before the close approach is assumed to be zero and the equation for the inclination after the passage is obtained by the following set of equations

$\left|\mathbf{C}_{\mathbf{0}}\right|=\mathrm{dV}_{\infty}\left(\begin{array}{l}(\sin \beta \sin \delta-\cos \beta \cos \delta \sin \gamma)^{2}+ \\ +\left(\frac{\mathrm{V}_{2}}{\mathrm{~V}_{\infty}}+\cos \alpha \cos \delta \cos \gamma-\cos \beta \sin \alpha \sin \delta-\cos \delta \sin \alpha \sin \beta \sin \gamma\right)^{2}\end{array}\right)^{1 / 2}$

$\mathrm{C}_{\mathrm{oZ}}=\mathrm{dV}_{\infty}\left(\frac{\mathrm{V}_{2}}{\mathrm{~V}_{\infty}}+\cos \alpha \cos \delta \cos \gamma-\cos \beta \sin \alpha \sin \delta-\cos \delta \sin \alpha \sin \beta \sin \gamma\right)(21)$

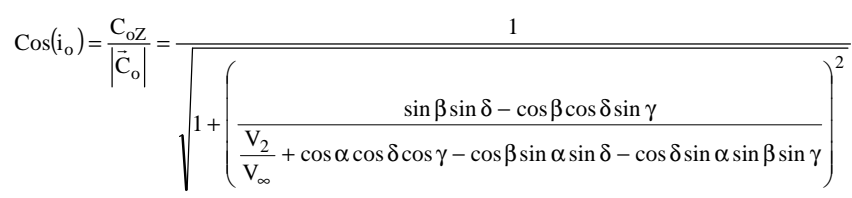

Where $\mathbf{C}_{\mathbf{0}}$ is the final angular momentum and $\mathrm{i}_{\mathrm{o}}$ is the final inclination. The subscript $\mathrm{Z}$ stands for the Z-component of the angular momentum. Equation 22 is a function of the two variables used as the control for the maneuver: the angle $\beta$ and the periapsis distance $r_{p}$. The values for the other variables are known: $V_{2}=1$, $\mathrm{V}_{\infty}$ is given by Eq. $6, \delta$ is given by Eq. $8, \gamma$ is given by Eq. 9 and $\alpha$ is obtained from $\alpha=\pi+\beta+\delta$. After all those considerations and also using the values $a=0.51 ; a_{0}=0.017$, the final equation for the inclination is given by 


$$
\begin{aligned}
& \mathrm{i}_{\mathrm{o}}=\operatorname{Cos}^{-1}\left[\left(1-\frac{0.822 \operatorname{Cos}[\beta] \operatorname{Sin}\left[3.234+\operatorname{Sin}^{-1}\left[\frac{1}{1+55.807 \mathrm{rp}}\right]\right]}{1+55.807 \mathrm{r}_{\mathrm{p}}}+\frac{0.822 \operatorname{Sin}[\beta] \operatorname{Sin}\left[3.234+\operatorname{Sin}^{-1}\left[\frac{1}{1+55.807 \mathrm{rp}}\right]\right] \operatorname{Tan}[\beta]}{1+55.807 \mathrm{r}_{\mathrm{p}}}\right.\right. \\
& \left.+0.822 \sqrt{1-\frac{1}{\left(1+55.807 \mathrm{r}_{\mathrm{p}}\right)^{2}}} \operatorname{Cos}\left[3.234+\operatorname{Sin}^{-1}\left[\frac{1}{1+55.807 \mathrm{r}_{\mathrm{p}}}\right]\right] \sqrt{1-\frac{\operatorname{Tan}^{2}[\beta]}{\left(1+55.807 \mathrm{r}_{\mathrm{p}}\right)^{2}\left(1-\frac{1}{\left(1+55.807 \mathrm{r}_{\mathrm{p}}\right)^{2}}\right)}}\right) \\
& \left(\frac{2.701 \operatorname{Sin}^{2}[\beta]}{\left(1+55.807 \mathrm{r}_{\mathrm{p}}\right)^{2}}+\left(1-\frac{0.822 \operatorname{Cos}[\beta] \operatorname{Sin}\left[3.234+\operatorname{Sin}^{-1}\left[\frac{1}{1+55.807 \mathrm{r}_{\mathrm{p}}}\right]\right]}{1+55.807 \mathrm{r}_{\mathrm{p}}}+\frac{0.822 \operatorname{Sin}[\beta] \operatorname{Sin}\left[3.234+\operatorname{Sin}^{-1}\left[\frac{1}{1+55.807 \mathrm{r}_{\mathrm{p}}}\right]\right] \operatorname{Tan}[\beta]}{1+55.807 \mathrm{r}_{\mathrm{p}}}\right.\right. \\
& \left.\left.\left.+0.822 \sqrt{1-\frac{1}{\left(1+55.807 \mathrm{r}_{\mathrm{p}}\right)^{2}}} \operatorname{Cos}\left[3.234+\operatorname{Sin}^{-1}\left[\frac{1}{1+55.807 \mathrm{r}_{\mathrm{p}}}\right]\right] \sqrt{1-\frac{\operatorname{Tan}^{2}[\beta]}{\left(1+55.807 \mathrm{r}_{\mathrm{p}}\right)^{2}\left(1-\frac{1}{\left(1+55.807 \mathrm{r}_{\mathrm{p}}\right)^{2}}\right)}}\right)^{2}\right]^{-1 / 2}\right]
\end{aligned}
$$

Figure 3 shows the three-dimensional view of this function. The function is not defined for all values of the angle $\beta$ and the periapsis distance $r_{p}$, because the equation has several square roots and inverse trigonometric functions that have limits of in their definitions.

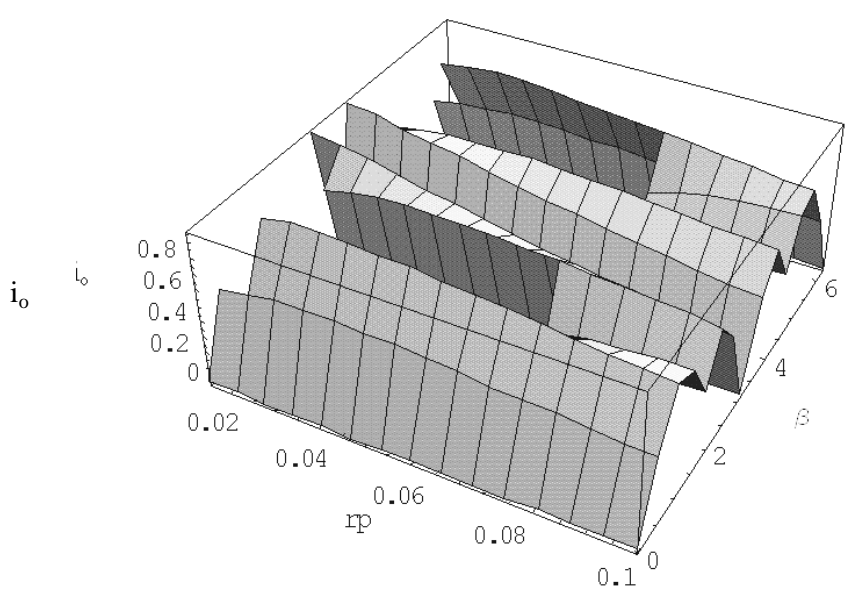

Figure 3. Inclination obtained by the swing-by (in radians) as a function of the angle $\beta$ (in radians, at the right side of the figure) and the periapsis distance $r p$ (in canonical units, at the left of the figure).

After that it is necessary to obtain the semi-major axis $\left(a_{f}\right)$ and the eccentricity $\left(\mathrm{e}_{\mathrm{f}}\right)$ of the orbit after the close approach. They are given by

$$
\begin{aligned}
& \mathrm{a}_{\mathrm{f}}=\frac{(1-\mu)}{2(1-\mu)-\mathrm{V}_{0}^{2}} \\
& \mathrm{e}_{\mathrm{f}}=\sqrt{1-\frac{\mathrm{C}_{0}^{2}}{(1-\mu) \mathrm{a}_{\mathrm{f}}}}
\end{aligned}
$$

With those quantities available, it is possible to obtain the impulses required to perform the maneuver in inclination of the spacecraft under two approaches: i) The standard maneuver of applying one impulse in the spacecraft when it passes by the intersection of the two orbits. For this maneuver the impulse required is given by (Chobotov, 1996)

$$
\Delta \mathrm{V}_{\mathrm{H}}=2 \sqrt{\frac{(1-\mu)}{\mathrm{a}_{0}}} \sin \left(\frac{\Delta \mathrm{i}}{2}\right)
$$

where $\Delta \mathrm{i}$ is the inclination desired for the maneuver.

ii) The proposed maneuver of sending the spacecraft to the Moon in a planar maneuver, to use the close approach with the Moon to change the inclination of the orbit with no cost, and then performing a two-impulsive maneuver to change the semi-major axis $\left(a_{f}\right)$ and the eccentricity $\left(e_{f}\right)$ of the orbit after the close approach back to their original values a and zero. This bi-impulsive maneuver has a cost giving by

$$
\Delta \mathrm{V}_{3}=\left|\sqrt{\frac{2(1-\mu)}{\mathrm{a}_{0}}-\frac{(1-\mu)}{\mathrm{a}_{\mathrm{t}}}}-\sqrt{\frac{(1-\mu)}{\mathrm{a}_{0}}}\right|
$$

where $a_{t}=\frac{a_{f}\left(1+e_{f}\right)+a_{0}}{2}$.

So, the total velocity increment is given by

$$
\Delta \mathrm{V}_{\mathrm{t}}=\Delta \mathrm{V}_{1}+\Delta \mathrm{V}_{2}+\Delta \mathrm{V}_{3}
$$

\section{Results}

Several simulations were performed with the equations shown above. Figure (4) shows the difference in fuel consumption $\left(\Delta \mathrm{V}_{\mathrm{t}}-\Delta \mathrm{V}_{\mathrm{H}}\right)$, measured in canonical units of increment of velocity to be applied in the spacecraft ( 1 canonical unit is equal to 1.023 $\mathrm{km} / \mathrm{s}$ ) as a function of the angle $\beta$, in radians, for three values of the periapsis distance $0.0048(1845 \mathrm{~km}), 0.0524(20142 \mathrm{~km})$ and 0.1000 $(38440 \mathrm{~km})$. It is visible that the difference in fuel consumption decreases with this variable, what is expected because the effects of the swing-by increases when the spacecraft is passing closer to the Moon and the inclination change is higher, increasing the savings of the maneuver that uses the swing-by. This is confirmed by Fig. 5, 
which shows the inclination change, in radians, obtained by the swing-by maneuver. Another property shown by this figure is that the interval of definition of the function increases when the value of the periapsis distance increases. In particular, it is interesting to note that when the periapsis distance goes from 0.0048 to 0.0524 two new regions of savings for the maneuver proposed appears, close to $\beta=1$ and $\beta=5$. It means that a very careful analysis of those parameters has to be made before making a final decision of a practical maneuver.

$$
\Delta \mathrm{V}_{\mathrm{t}}-\Delta \mathrm{V}_{\mathrm{H}} \Delta \mathrm{V}_{\mathrm{t}}-\Delta \mathrm{V}_{\mathrm{H}}
$$

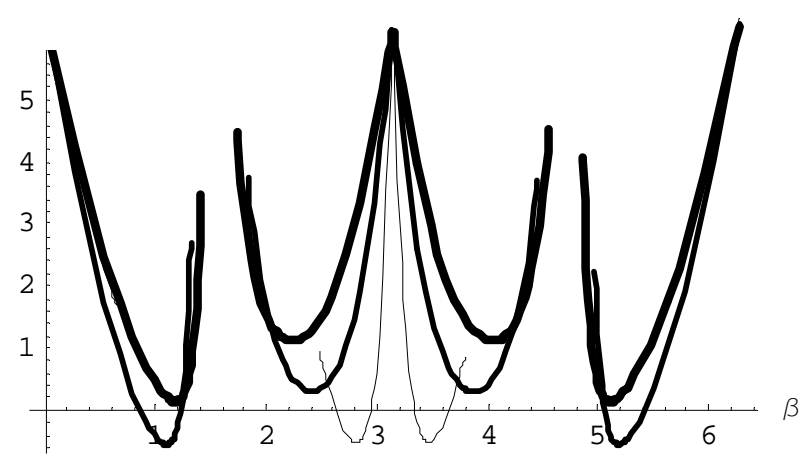

Figure 4. $\Delta \mathrm{V}_{t}-\Delta \mathrm{V}_{\mathrm{H}}$ (canonical units) as a function of $\beta$ (in radians) for $\mathrm{a}_{0}$ $=0.017$ and $a=0.51$. The darkest line represents the results for $r_{p}=0.1$, the intermediate line represents the results for $r_{p}=0.0524$ and the thinnest line represents the results for $r_{p}=0.0048$.

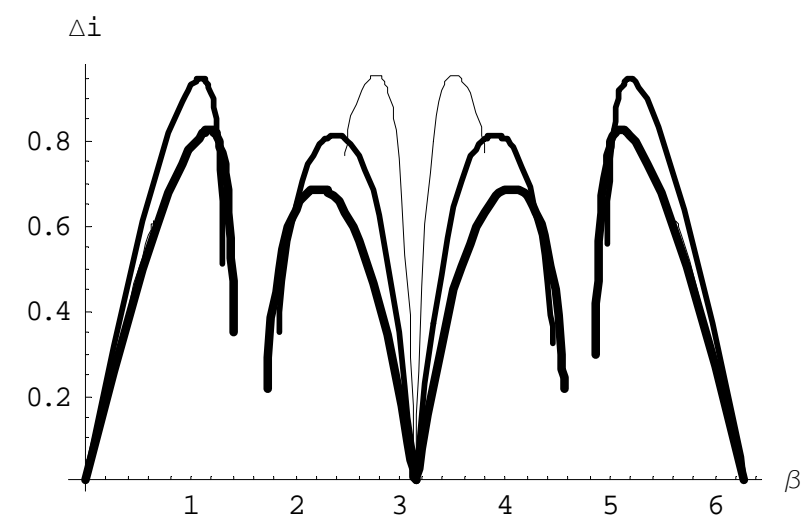

Figure 5 . Inclination change (in radians) as a function of $\beta$ (in radians) for $a_{0}=0.017$ and $a=0.51$. The darkest line represents the results for $r_{p}=0.1$, the intermediate line represents the results for $r_{p}=0.0524$ and the thinnest line represents the results for $r_{p}=\mathbf{0 . 0 0 4 8}$.

Next, the effect of the semi-major axis of the transfer orbit from the Earth to the Moon is studied. Figure 6 shows the same difference in fuel consumption $\left(\Delta \mathrm{V}_{\mathrm{t}}-\Delta \mathrm{V}_{\mathrm{H}}\right)$ as a function of $\beta$ for $\mathrm{a}_{0}=0.017$ and $r_{p}=0.0048$ and considering three values for a: 0.51 (the minimum to achieve the orbit of the Moon), 0.56 and 0.61 . It is clear that the savings of the swing-by maneuver increase when the value of this variable increases. Simulations with a larger number of values for this variable show that this behavior stays the same until the infinity. The reason is that the increase in fuel consumption in the first impulse required to obtain a larger value of the semi-major axis is small when compared to the gains obtained with a swing-by performed with a higher approaching velocity.

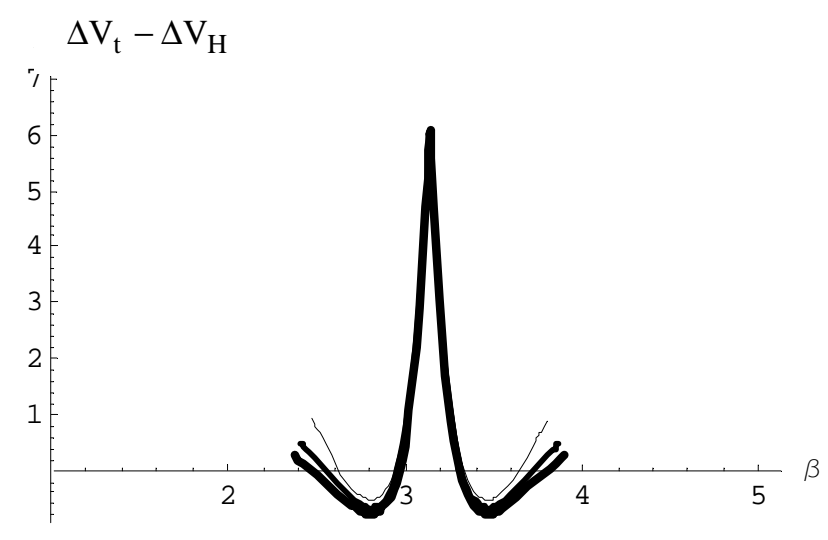

Figure 6. $\Delta \mathrm{V}_{\mathrm{t}}-\Delta \mathrm{V}_{\mathrm{H}}$ (in canonical units) as a function of $\beta$ (in radians) for $a_{0}=0.017$ and $r_{p}=0.0048$. The darkest line represents the results for $a=$ 0.61 , the intermediate line represents the results for $a=0.56$ and the thinnest line represents the results for $a=0.51$.

Then, the effects of varying the initial orbit of the spacecraft around the Earth are considered. Figure 7 shows the difference in fuel consumption $\left(\Delta \mathrm{V}_{\mathrm{t}}-\Delta \mathrm{V}_{\mathrm{H}}\right)$ as a function of $\beta$ for $\mathrm{r}_{\mathrm{p}}=0.0048$ $(1845 \mathrm{~km}), \mathrm{a}=0.51(196044 \mathrm{~km})$ and three values for the semimajor axis of the initial orbit: $0.017(6535 \mathrm{~km}), 0.024(9226 \mathrm{~km})$ and $0.030(11532 \mathrm{~km})$. The results show that the advantages of the swing-by maneuver increases when the value of the initial semimajor axis decreases. This result is expected because the costs of the inclination change increase very much when the initial orbit gets closer to the Earth.

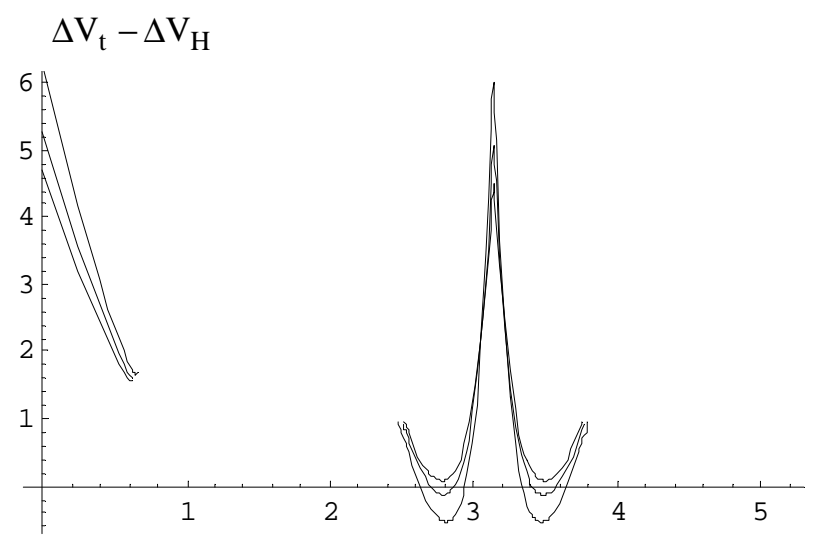

Figure 7. $\Delta \mathrm{V}_{t}-\Delta \mathrm{V}_{\mathrm{H}}$ (in canonical units) as a function of $\beta$ (in radians) for $r_{p}=0.0048$ and $a=0.51$. The bottom line represents the results for $a_{0}=$ 0.017 , the intermediate line represents the results for $a_{0}=0.024$ and the superior line represents the results for $\mathrm{a}_{0} \mathbf{= 0 . 0 3 0}$.

\section{Conclusions}

A set of analytical equations is used to describe the swing-by in three dimensions and to evaluate the variation in the inclination of the orbit of a spacecraft that is passing by the Moon. Then, it is possible to compare analytically two schemes to make an orbital maneuver that has only an inclination change in the orbit as its goal: i) the standard single impulse maneuver that applies the impulse in the crossing point between the two orbits and, ii) a three-impulsive transfer that applies the first impulse to send the spacecraft to the Moon, uses the gravity field of the Moon to perform a zero cost inclination change maneuver, and then makes two more impulses to accomplish a planar Hohmann transfer from the orbit that is 
obtained after the swing-by and the desired final orbit. The results showed that in several circumstances this second maneuver can be more economical than the standard maneuver, if a proper choice of the periapsis position is made. It is also showed that this maneuver is more economical if: i) the initial parking orbit is close to the Earth, ii) the semi-major axis of the transfer orbit from the Earth to the Moon tends to infinity, iii) the periapsis of the close approach to the Moon has the smallest possible value. Then, this research can be used to design maneuvers for low Earth orbit satellites that requires larger inclination changes.

\section{Acknowledgment}

The author is grateful to the Foundation to Support Research in the São Paulo State (FAPESP) for the research grant received under Contract 2000/14769-4 and to CNPq (National Council for Scientific and Technological Development) - Brazil for the contract 300221/95-9.

\section{References}

Broucke, R.A. \& A.F.B.A. Prado (1993). "Jupiter Swing-By Trajectories Passing Near the Earth", Advances in the Astronautical Sciences, Vol. 82, No 2, pp. 1159-1176.

Broucke, R.A. (1988). "The Celestial Mechanics of Gravity Assist", AIAA Paper 88-4220.

Byrnes, D.V. \& L.A. D'Amario (1982) "A Combined Halley Flyby Galileo Mission," AIAA paper 82-1462. In: AIAA/AAS Astrodynamics Conference, San Diego, CA, Aug.

Chobotov, V. A. (1996) Orbital motion - Second Edition. Reston: American Institute of Aeronautics and Astronautics, 1996.

D'Amario, L.A., D.V. Byrnes \& R.H Stanford. (1981). "A New Method for Optimizing Multiple-Flyby Trajectories, " Journal of Guidance, Control, and Dynamics, Vol. 4, No 6, pp. 591-596.

D'Amario, L.A., D.V. Byrnes \& R.H. Stanford (1982). "Interplanetary Trajectory Optimization with Application to Galileo," Journal of Guidance, Control, and Dynamics, Vol. 5, No. 5, pp. 465-471.

Dunham, D. \& S. Davis (1985). "Optimization of a Multiple LunarSwing by Trajectory Sequence," Journal of Astronautical Sciences, Vol. 33, No. 3, pp. 275-288.

Efron, L., D.K. Yeomans \& A.F. Schanzle (1985). "ISEE-3/ICE Navigation Analysis," Journal of Astronautical Sciences, Vol. 33, No. 3, pp. 301-323.
Farquhar, R., D. Muhonen \& L.C Church (1985). "Trajectories and Orbital Maneuvers for the ISEE-3/ICE Comet Mission", Journal of Astronautical Sciences, Vol. 33, No. 3, pp. 235-254.

Farquhar, R.W. \& D.W. Dunham (1981). "A New Trajectory Concept for Exploring the Earth's Geomagnetic Tail", Journal of Guidance, Control and Dynamics, Vol. 4, No. 2, pp. 192-196.

Felipe, G. \& A.F.B.A. Prado (1999). "Classification of Out of Plane Swing-by Trajectories". Journal of Guidance, Control and Dynamics, Vol. 22, No. 5, pp. 643-649.

Felipe, G. and Prado, A.F.B.A. (2000). "Study of the Inclination Change in Three-Dimensional Swing-By Trajectories". $22^{\text {nd }}$ International Symposium on Space Technology and Science, pp. 60 (00-j-02). Congress realized in 28-May to 04-June, 2000, Morioka, Japan.

Flandro, G. (1966). "Fast Reconnaissance Missions to the Outer Solar System Utilizing Energy Derived from the Gravitational Field of Jupiter," Astronautical Acta, Vol. 12, No. 4.

Marsh, S.M. \& K.C. Howell (1988). "Double Lunar Swing by Trajectory Design," AIAA paper 88-4289.

Muhonen, D., S. Davis, \& D. Dunham (1985). "Alternative GravityAssist Sequences for the ISEE-3 Escape Trajectory," Journal of Astronautical Sciences, Vol. 33, No. 3, pp. 255-273.

Prado, A.F.B.A. \& R.A. Broucke (1994). "A Study of the Effects of the Atmospheric Drag in Swing-By Trajectories," Journal of the Brazilian Society of Mechanical Sciences, Vol. XVI, pp. 537-544.

Prado, A.F.B.A. \& R.A. Broucke (1995). "A Classification of Swing-By Trajectories using The Moon". Applied Mechanics Reviews, Vol. 48, No. 11, Part 2, November, pp. 138-142.

Prado, A.F.B.A. (1993). "Optimal Transfer and Swing-By Orbits in the Two- and Three-Body Problems", Ph.D. Dissertation, Dept. of Aerospace Engineering and Engineering Mechanics, Univ. of Texas, Austin, TX.

Prado, A.F.B.A. (1996). "Powered Swing-By". Journal of Guidance, Control and Dynamics, Vol. 19, No. 5, pp. 1142-1147.

Prado, A.F.B.A. (1997). "Close-approach Trajectories in the Elliptic Restricted Problem", Journal of Guidance, Control, and Dynamics, Vol. 20, No. 4, pp. 797-802.

Prado, A.F.B.A. (2000), "An Analytical Description of the Close Approach Maneuver in Three Dimensions". 51 ${ }^{\text {th }}$ International Astronautical Congress (IAF-00-A.5.05). Rio de Janeiro, Brazil, 02-06 October, 2000.

Striepe, S.A. \& R.D. Braun (1991). "Effects of a Venus Swing by Periapsis Burn During an Earth-Mars Trajectory," The Journal of the Astronautical Sciences, Vol. 39, No. 3, pp. 299-312..

Swenson, B.L. (1992). "Neptune Atmospheric Probe Mission", AIAA Paper 92-4371.

Weinstein, S.S. (1992). "Pluto Flyby Mission Design Concepts for Very Small and Moderate Spacecraft", AIAA Paper 92-4372. 